\title{
Remarkable virus diversity and organized community in filter-feeding oysters varied from those of the ocean virome
}

Jing-Zhe Jiang ( $\nabla_{\text {jingzhejiang@gmail.com ) }}$

Ministry of Agriculture, Chinese Academy of Fishery Sciences

Yi-Fei Fang

Shanghai Ocean University

Hong-Ying Wei

Guangdong Magigene Biotechnology Co., Ltd

Peng Zhu

Shanghai Ocean University

Min Liu

Shanghai Ocean University

Li-Ling Yang

Tianjin Agricultural University

\section{Ying-Xiang Guo}

Tianjin Agricultural University

\section{Tao Jin}

Guangdong Magigene Biotechnology Co., Ltd

\section{Mang Shi}

Sun Yat-sen University

\section{Tuo Yao}

Ministry of Agriculture, Chinese Academy of Fishery Sciences

Jie Lu

Ministry of Agriculture, Chinese Academy of Fishery Sciences

Ling-Tong Ye

Ministry of Agriculture, Chinese Academy of Fishery Sciences

Shao-Kun Shi

Shenzhen Fisheries Development Research Center

\section{Meng Wang}

Bureau of Agriculture and Rural Affairs of Conghua District

\section{Ming Duan}

Chinese Academy of Sciences

\section{Dian-Chang Zhang}




\section{Research Article}

Keywords: Crassostrea hongkongensis, Bivalve, Mollusk, Metagenome, Circoviridae, Viral-Like Particle Enrichment, South China, Multiple Displacement Amplification

Posted Date: February 11th, 2022

DOI: https://doi.org/10.21203/rs.3.rs-1344035/v1

License: (a) (i) This work is licensed under a Creative Commons Attribution 4.0 International License. Read Full License 


\section{Abstract}

\section{Background:}

Viruses play critical roles in the marine environment because of their interactions with an extremely broad range of potential hosts. Many studies of viruses in seawater have been published, but viruses that inhabit marine animals have been largely neglected. Oysters are keystone species in coastal ecosystems, but as filter-feeding bivalves with very large roosting numbers and species co-habitation, it is not clear what role they play in marine virus transmission and coastal microbiome regulation.

\section{Results:}

Here we report a Dataset of Oyster Virome (DOV) that contains 728,784 nonredundant viral operational taxonomic unit (vOTU) ( $\geq 800 \mathrm{bp}$ ) contigs and 3,473 high-quality viral genomes, enabling the first comprehensive overview of both DNA and RNA viral communities in oysters (Crassostrea hongkongensis). We discovered tremendous diversity among novel viruses that inhabit oysters using multiple approaches, including reads recruitment, vOTUs, and high-quality virus genomes. Our results show that these viruses are very different from viruses in the oceans or other habitats. In particular, the high diversity of novel circoviruses that we found in oysters indicates that oysters may be potential hotspots for circoviruses. Notably, the viruses that were enriched in oysters are not random but are well organized communities that can respond to changes in the health state of the host and the external environment at both compositional and functional levels.

\section{Conclusions:}

In this study, we generated a first knowledge landscape of the oyster virome, which has increased the number of known oyster-related viruses by tens of thousands of times. Our results suggest that oysters provide a unique habitat that is different from that of seawater, and highlight the importance of filterfeeding bivalves for marine virus exploration as well as their essential but still invisible roles in regulating marine ecosystems.

\section{Background}

As the most abundant biological entities on Earth, viruses can hijack organisms from every branch of the tree of life. They play critical roles in host mortality, metabolism, physiology, and evolution, impacting marine biogeochemical cycling and shaping the Earth's microbiomes (Fuhrman, 1999; Wommack and Colwell, 2000; Suttle, 2007). Culture-independent next-generation sequencing technologies have recently been used to explore the tremendous diversity of the virosphere from multiple samples (Paez-Espino et al., 2016; Shi et al., 2016; Shi et al., 2018; Camarillo-Guerrero et al., 2021; Nayfach et al., 2021). Among the findings, progress in the discovery of marine viruses (mainly phages of marine bacteria) is particularly impressive (Brum et al., 2015; Wolf et al., 2020), including the creation of a global ocean DNA virome 2.0 
(GOV 2.0) dataset, which contains 195,728 viral populations detected in 145 seawater samples collected worldwide (Gregory et al., 2019).

Many studies have focused on free viruses in seawater, whereas viruses in marine animals have been largely neglected. Marine animals are teeming with viruses that inhabit the hosts' surfaces, body spaces, and blood (Scanes et al., 2021). Virome of marine animals form connections with their host, which is vital to the interaction of the microbe community both in and outside the host's body. Among these, bivalves, such as oysters and mussels, may play a particularly important role to the coastal and estuarine environment. That is because bivalves are sedentary and thus imposing a stable and lasting ecological effect to a given area. The population characteristics of bivalves, including very large roosting numbers and species co-habitation, provide the perfect setting for viral transmission with the flow of ocean currents. Most importantly, as filter-feeding animals, they can draw up to $5 \mathrm{~L}$ of water per hour through their gills and concentrate suspended microbes and particles by factors of a thousand to a hundred thousand times their seawater concentrations (Bedford, 1978; Olalemi et al., 2016). Indeed, the enrichment of human enteric viruses (Newell et al., 2010) and mimiviruses (Andrade et al., 2015) in oyster gill or gut tissues is clearly a filter-feeding effect. However, the circulatory system of bivalves is open because, unlike vertebrates, they have no vessels to hold blood. In bivalves, the blood (hemolymph) flows freely throughout the body cavities, and therefore it is interesting to speculate what the virus communities in bivalves look like. For example, whether bivalves provide a similar environment or a unique habitat for marine viruses and whether bivalves have the potential to spread viruses and regulate coastal microbial communities are important questions that are yet to be answered.

Oysters, in family Ostreidae, which widely distributed in the intertidal zone all over the world, are the most highly produced seafood in the world. China is the largest oyster producer, accounting for $85.3 \%$ of the world's total production (FAO, 2019). As keystone species, oysters provide essential benefits to coastal and estuarine ecosystems, improving water quality and providing a critical habitat for various organisms (Zhang et al., 2012; Powell et al., 2018). Here we report an extensive Dataset of Oyster Virome (DOV) that consists of 54 sequencing libraries from different tissues, sampling sites, and sampling times of Crassostrea hongkongensis, the most farmed species of oyster along the South China coast. We used viral-like particle (VLP) enrichment and targeted amplification strategies, and built a knowledge landscape of the oyster virome community, its function, and influencing factors of both RNA and DNA viruses, which provides a good foundation to address questions about the connections between bivalves and marine viruses.

\section{Material And Methods}

\subsection{Oyster sampling}

The oyster samples collected in this study span five years, from June 2014 to July 2019 . We divided the samples into nine time batches according to the chronological order. In addition, all the samples were divided into four other groups: four amplification groups based on the amplification methods (whole 
genome amplification (WGA), whole transcriptome amplification (WTA), reverse transcription and WGA (RT-WGA), and double-stranded DNA (dsDNA)); two tissue groups based on tissue origin (mixed tissues and hemolymph of adults); two status groups based on health status (diseased and moribund), and seven Site groups based on sampling sites (BH, HD, LJ, SZ, TS, YJ, and ZH) (Fig. 1E). In total, we constructed 54 sequencing libraries with 35 samples. For more information, see Table S1.

For eight of the nine time batches, the tissues without the gonad from three oysters were mixed into one sample; the seventh batch was the exception. The first batch, dCh, contained samples of dying adult $C$. hongkongensis collected from an oyster farming area in Beihai (BH) of Guangxi Province in June 2014. The second batch had two groups, YJd and YJr, and contained samples of healthy adult $C$. hongkongensis collected in September 2015 from an oyster farming area in Yangjiang (YJ), Guangdong. The downstream amplification method for YJd was WGA (to detect mainly DNA virus genomes) and for YJr it was WTA (to detect mainly RNA virus genomes). The third batch had eight groups, LJd, LJr, QZd, $\mathrm{QZr}$, TWd, TWr, ZHd, and ZHr, and contained healthy adult $C$. hongkongensis collected from oyster farming areas in the Qinzhou area (QZ) of Beihai (BH), Tanwei area (TW) of Huidong (HD), Zhuhai (ZH), and Lianjiang (LJ) of Guangdong Province in November 2015. The fourth batch had two groups, SZd and $\mathrm{SZr}$, and contained healthy adult $C$. hongkongensis collected from the Shenzhen (SZ) oyster farming area in Guangdong in April 2016. (The letters " $d$ " and " $r$ " indicate WGA and WTA, respectively, in the third and fourth batches.) The fifth batch, ML, contained healthy adult $C$. hongkongensis collected from SZ in May 2016. The downstream amplification method for ML was RT-WGA (to detect both DNA and RNA virus genomes). The sixth batch, $\mathrm{BH}$, contained moribund adult $C$. hongkongensis collected from $\mathrm{BH}$ in July 2016. The seventh batch had nine groups. GX, K1ZY, K2ZY, T2S, T4S, T5S, T6S, T8S, and ZH, and contained adult $C$. hongkongensis that were separately collected from $\mathrm{BH}$ in Guangxi Province, Kaozhouyang (K\#ZY) of Huidong (HD), Taishan (T\#S), and Zhuhai (ZH) in Guangdong Province in May 2017. K1ZY, K2ZY, and T8S contained healthy adult $C$. hongkongensis, and the other groups contained moribund adult $C$. hongkongensis. The method of sampling in this batch was different from the method used for all the other batches. A 1-mL syringe was used to draw hemolymph from the pericardial cavity of oysters, and samples from 5-8 oysters were mixed into one sample. The eighth batch, os, contained adult $C$. gigas collected in July 2018. The samples in these eight batches were collected and preserved by the South China Sea Fisheries Research Institute (Guangdong, China). The ninth batch had two groups, $\mathrm{HSd}$ and $\mathrm{HSr}$, and contained healthy adult $C$. hongkongensis purchased from the Huangsha Aquatic Product Market in Guangzhou (GZ), Guangdong, in July 2019; their original farming location was ZH. The samples in this batch were collected and preserved by Guangdong Magigene Technology Co., Ltd (Guangzhou, China). All the samples were quickly frozen in liquid nitrogen, temporarily stored during transportation, and placed in an ultra-low temperature freezer at $-80^{\circ} \mathrm{C}$ for long-term storage.

\subsection{VLP enrichment}

All 35 samples were processed to enrich for VLPs as described by Wei et al. (2017) and using the online protocols (dx.doi.org/10.17504/protocols.io.m4yc8xw). First, $500 \mathrm{mg}$ of mixed tissue, or 14-34 mg spat 
mixture, was dissected and ground to powder in liquid nitrogen. The powder was further homogenized in approximately 2- 5 volumes of sterile SB buffer $(0.2 \mathrm{M} \mathrm{NaCl}, 50 \mathrm{mM}$ Tris- $\mathrm{HCl}, 5 \mathrm{mM} \mathrm{CaCl} 2,5 \mathrm{mM} \mathrm{MgCl}$, $\mathrm{pH}$ 7.5). After three rounds of freezing and thawing, the pellets were resuspended entirely in 10 volumes of pre-cooled SB buffer. For the hemolymph sample, $10 \mathrm{~mL}$ hemolymph was mixed with an equal volume of $2 \times \mathrm{SB}$ buffer, then directly subjected to three rounds of freezing and thawing. The following steps were the same for the tissue, spat, and hemolymph samples. All the samples were centrifuged at $1,000,3,000$, $5,000,8,000,10,000$, and $12,000 \times \mathrm{g}$ for $5 \mathrm{~min}$ each at $4^{\circ} \mathrm{C}$ using a $3 \mathrm{~K} 30$ centrifuge (Sigma, Osterode am Harz, Germany), and the supernatants were retained. Cell debris, organelles, and bacterial cells were further removed using a Millex-HV $0.22 \mu \mathrm{m}$ filter. The filtrates were transferred to ultracentrifuge tubes containing $28 \%(\mathrm{w} / \mathrm{w})$ sucrose using a syringe. The tubes were transferred to an ice bath for $10 \mathrm{~min}$ before centrifugation in a Himac CP 100WX ultracentrifuge (Hitachi, Tokyo, Japan) at 300,000 $\times \mathrm{g}$ for 2 hr. Supernatants were discarded and the precipitates were fully resuspended in $720 \mu$ of water, $90 \mu \mathrm{l} 10 \times$ DNase I Buffer, $90 \mu \mathrm{l} \mathrm{DNase} \mathrm{I}(1 \mathrm{U} / \mu \mathrm{l})$, and incubated at $37^{\circ} \mathrm{C}$ with shaking for $60 \mathrm{~min}$, followed by storage overnight at $4^{\circ} \mathrm{C}$, and transfer to 2 -ml centrifuge tubes.

\subsection{Viral nucleic acid extraction and amplification}

Total nucleic acid was extracted from the VLPs using an HP Viral DNA/RNA Kit (R6873; Omega Bio-Tek, Norcross, USA); carrier RNA was not used, to avoid potential interference with sequencing results. A Qubit $^{\text {TM }}$ dsDNA HS Assay Kit (Q32851) and Qubit ${ }^{\text {TM }}$ RNA HS Assay Kit (Q32855) (Thermo Fisher Scientific, Waltham, USA) were used to quantify the concentrations of dsDNA and RNA separately.

Virome studies are highly reliant on amplification because the viral biomass in natural samples is very low (Polson et al., 2011; Bar-On et al., 2018). Because most amplification methods introduce bias, it is challenging to study viromic data quantitatively at present (Parras-Moltó et al., 2018; Fan et al., 2021). Here, a REPLI-g Cell WGA and WTA Kit (150052, Qiagen, Hilden, Germany), which is based on the multiple displacement amplification (MDA) method, was used to uniformly amplify the genomes (WGA) and transcriptomes (WTA) (Hosono et al., 2003; Pan et al., 2013; Picher et al., 2016). MDA has many significant advantages over other amplification methods, such as replicating up to $70 \mathrm{~kb}$, more even coverage, and 1000-fold higher fidelity than Taq polymerase amplification (Hosono et al., 2003; Stepanauskas et al., 2017), which make MDA widely used in virome studies.

To better compare the RNA and DNA virus communities, we used WGA and WTA methods to construct libraries in four batches of mixed tissues, which accounted for $70 \%(38 / 54)$ of all libraries (Table S1). RTWGA is a modified protocol that simultaneously amplifies DNA and RNA (Wei et al., 2018b; Li et al., 2019). In this study, 14 libraries were constructed based on RT-WGA, including hemolymph and mixed tissue samples (Table S1). The steps for the WGA, WTA, and RT-WGA were according to the online protocols (dx.doi.org/10.17504/protocols.io.m5vc866). For WTA, there is a "DNA wipeout" step before reverse transcription that aims to remove DNA altogether, but this step is not part of the WGA and RT-WGA protocols. Compared with WTA and RT-WGA, the WGA protocol skips the reverse transcription reaction to 
avoid amplifying RNA in the downstream reaction. In addition, two other samples were directly subjected to random shotgun library preparation using a Nextera XT DNA Library Preparation Kit (Illumina) following the standard manufacturer's protocol. Because of the limited data quality and sample number, these two libraries were not included in the following diversity analysis.

\subsection{Library construction and sequencing}

Amplified DNA was quantified by gel electrophoresis and Nanodrop 2000 spectrophotometer (Thermo Fisher Scientific) and randomly sheared by ultrasound sonication (Covaris M220) to produce fragments $\leq 800$-bp long. The sticky ends were repaired, and adapters were added using T4 DNA polymerase (M4211, Promega, USA), Klenow DNA Polymerase (KP810250, Epicentre), and T4 polynucleotide kinase (EK0031, Thermo Fisher Scientific, USA). Fragments of 300-800 bp were collected after electrophoresis. After amplification, libraries were pooled and subjected to $150 \mathrm{bp}, 250 \mathrm{bp}$, or $300 \mathrm{bp}$ paired-end sequencing on Novaseq 6000, HiSeq X ten, and Miseq platforms (Illumina, USA). Considering the RT-WGA libraries were likely to have higher virus diversity than the WGA and WTA libraries (Wei et al., 2018a), they were sequenced with higher depth and also produced better assembly results (Table S1).

\subsection{Virus detection and quantification based on reference viral genomes}

Instead of using the traditional read alignment tools such as BLAST, BWA, and Bowtie2, we used FastViromeExplorer (Tithi et al., 2018), which was developed for fast and accurate virus detection and quantification in metagenomics data. FastViromeExplorer filters the alignment results based on minimal coverage criteria and the minimal number of mapped reads and accurately reports virus types and relative abundances. The Kallisto (version 0.43.1) method, integrated with FastViromeExplorer, was used with the default settings to map clean reads against three reference databases: the National Center for Biotechnology Information (NCBI) Reference Sequence (RefSeq) database, Global Ocean Virome database (GOV; Roux et al., 2016), and the Integrated Microbial Genome/Virus (IMG/VR) system, separately, to generate a reference abundance table. The RefSeq database contained 14,042 viral genomes or genome segments (update till 30 May 2019), GOV (Roux et al., 2016) includes 298,383 epipelagic and mesopelagic viral contigs, and IMG/VR contains 125,842 metagenomic viral contigs of the set of sequences collected from the Joint Genome Institute "earth virome" study (Paez-Espino et al., 2016).

\subsection{Virus detection and quantification based on de novo assembly (vOTU annotation)}

High-quality clean reads were generated using Fastp (version 0.20.0) (Chen et al., 2018), (options: -correction, --trim_poly_g, -trim_poly_x, --overrepresentation_analysis, -trim_front1 $=16$, -trim_tail1 $=2$, and -- 
length_required=50) and reads that matched the Illumina sequencing adapters were removed (option: -detect_adapter_for_pe). The clean reads in libraries that were in the same assembly group were pooled and assembled using Megahit (version 1.2.9) (Li et al., 2015) with the default settings. Only contigs longer than $800 \mathrm{bp}$ were kept. To detect low abundant contigs, clean reads that did not map back to the first round of assembled contigs were reassembled for two additional rounds, then all remaining reads were pooled and assembled together. Contigs from all four assembly rounds were pooled, and clustered at $97 \%$ global average nucleotide identity with at least $90 \%$ overlap of the shorter contig using cd-hit-est (version 4.8.1) (options: -aS 0.9 -c $0.97-\mathrm{G} 1$-M 0 -T 0 -g 1), resulting in 3,347,421 nonredundant contigs (Fig. 1A).

Diamond (Buchfink et al., 2015) is a state-of-the-art method that can annotate sequences with high precision and speed. Compared with BLAST searches against virus only databases, BLAST searches against the NCBI nr database of nonredundant protein sequences can significantly lower the number of false positive results (Nouri et al., 2018; Yao et al., 2020). However, BLAST has relatively low accuracy for short fragments (Jiang et al., 2011) and it cannot be used for sequences that have no similarity. Although, it is possible to dig deeper into the "dark matter" by combining multiple virus mining tools, such as CheckV (Nayfach et al., 2021) and VirSorter2 (Guo et al., 2021), identifying and classifying suspected viral sequences is challenging because there is a lack of adequate credible annotations (Handley et al., 2019). Given these challenges, the nonredundant contigs were annotated only using Diamond (version 0.9.24.125, options: -e 1e-10, -max-target-seqs 50) against the NCBI nr database (as of 11 July 2019). Among them, $728,784(21.77 \%)$ of the total contigs were annotated as viral origin (i.e., vOTUs). Among them, $7.68 \%$ were Eukaryota, $0.34 \%$ were Archeae, $21.59 \%$ were bacteria, and $0.82 \%$ were unclassified cellular organisms, and $47.89 \%$ unknown origin (Fig. 1A). FastViromeExplorer was used with default settings to map the clean reads against the vOTU contigs to obtain the vOTUs abundance table.

\subsection{Viral genome integrity, taxonomy, and auxiliary metabolic genes analysis}

The viral genome completeness of assigned contigs was tested using CheckV (version 0.7.0) and its associated database (Nayfach et al., 2021). After removing false positive contigs that matched more host genes than viral genes, 3,473 nearly complete viral genomes were obtained.

Three methods (Diamond, vContact2, and PhaGCN) were used to determine the taxonomy of the viral contigs at the family level. Diamond annotations were further processed using two scripts (daa2rma and rma2info) in MEGAN6 (Huson et al., 2016) with default parameters, and parsed to taxonomy annotations. The advantage of Diamond is that there is no minimum length requirement for query sequences; however, it has three drawbacks: low accuracy, low annotation rates, and inaccurate taxonomy of NCBI. PhaGCN is a novel semi-supervised learning model that combines the strengths of a BLAST-based model and learning-based model using a knowledge graph (Shang et al., 2017). For comparison purposes, only vOTUs that were longer than $10 \mathrm{~kb}$ were analyzed using PhaGCN and vContact2 with default parameters. 
To mine the auxiliary metabolic genes (AMGs) from DOV, Vibrant v1.2.1 (Kieft and Anantharaman, 2020) was used. Salmon v1.5.2 (Patro et al., 2017) was used with default settings to map clean reads against the AMG dataset to obtain the AMGs abundance table.

\subsection{Viral contamination assessment}

The experimental preparation for viromic sequencing involves the use of various reagents, many of which have been proved to carry contaminated viral sequences of unknown origin (Holmes, 2019). The extent of viral contamination in common laboratory components, especially viruses with small single-stranded DNA (ssDNA) genomes, has been reported previously (Asplund et al., 2019; Ashleigh et al., 2021).

To assess the viral contaminant level in this study, all the $3,347,421$ nonredundant contigs ( $\geq 800 \mathrm{bp}$; not only viral contigs) in DOV were used as queries in a BLASTN search (with the parameters set as 95\% identity and $95 \%$ query coverage) against the approximately 500 contaminant viral sequences reported by Asplund et al. (2019) and Ashleigh et al. (2021). We found very little evidence of viral contamination; no sequences matched with $100 \%$ identity, no expected circoviruses or RNA viruses were detected, and most of the alignments were with dsDNA phages (Additional file 1). The 3,473 near-complete viral genomes were used as queries in the same BLASTN search, but no matches were found. We also used Salmon (v1.5.2) to map all the clean reads in the DOV libraries to the contaminant viral sequences. The mapping rates for most of these libraries were $<0.01 \%$ (Additional file 2 ), which is consistent with the BLASTN results.

\subsection{Viral community and statistical analysis}

In this study, the FPKM (Fragments Per Kilobase per Million) value was used to represent the relative abundance of the reference viral genomes, vOTUs, and AMGs. On the basis of the FPKM-transformed abundance table, $\mathrm{R}$ and Excel were used to analyze the corresponding viral diversity and community structures. The vegan and ggplots $\mathrm{R}$ packages were used to calculate a-diversity indexes and plot the nonmetric multidimensional scaling (NMDS). Analysis of variance (ANOVA) and TukeyHSD were used to test the differences between groups with the significance level set at 0.05 . For the procrustes analysis, the characteristic axis coordinates of NMDS were extracted as the input of the procrustes function, and the protest function was used to perform the substitution test to evaluate the significance of the results.

\section{Result And Discussion}

\subsection{Overview of the Dataset of Oyster Virome (DOV)}

For this study, we collected 35 samples of mixed tissue or hemolymph from Crassostrea hongkongensis at nine time points from seven major oyster farming areas along the coast of South China (Fig. 1, Table S1). Fifty-four oyster virome libraries were constructed using three primary amplification methods (WTA, WGA, and RT-WGA) and sequenced (Table S1). A total of 3,347,421 nonredundant contigs ( $\geq 800 \mathrm{bp}$ ) were 
obtained after assembly. Among them, 728,784 (21.77\%) were annotated as viral origin by comprehensive blast (Fig. 1A), which we called the DOV. The viral contigs were assembled mainly from the RT-WGA libraries of hemolymph samples with higher sequencing coverages (Fig. 1B). Rarefaction curves (Fig. 1C) show that the sequencing depths were sufficient, and the vOTU numbers in the WTA libraries were the lowest among all the libraries.

Notably, the ratio of viral reads (mapping rate) varied a lot depending on the reference databases that were searched (Fig. 1E). The mapping rate of de novo assembled vOTUs $(29.81 \%)$ was much higher than the mapping rates of the RefSeq (NCBI viral reference genomes) $(3.50 \%)$ and the RefSeq plus two other public virus datasets (GOV and IMG/VR) (12.06\%) (Fig. 1E, Table S1). The higher mapping rates of vOTUs confirmed that the VLPs enrichment protocol was effective (Wei et al., 2018b; Liu et al., 2019), and filterfeeding oysters can efficiently accumulate environmental viruses. The low mapping rates of the reference genomes $(3.50 \%$ and $12.06 \%$ ) imply that the viruses found in oysters are largely unknown. To our knowledge, this is the biggest viral metagenomic dataset for any marine animal currently available.

\subsection{Viruses in Oysters}

Rosani et al. (2017 and 2019) assembled 26 novel and nearly complete RNA virus genomes from the public transcriptomic data of $C$. gigas and $C$. corteziensis, and Zhang et al. (2021) reported four new RNA virus genomic fragments from $C$. gigas, which were recovered from a virome survey of marine invertebrates. Another 33 novel RNA viruses were identified from mixed bivalve samples (including two oyster species $C$. hongkongensis and $C$. ariakensis) (Shi et al., 2016). Although we recovered 4,958 RNA vOTUs from $C$. hongkongensis, they accounted for only $0.68 \%$ of all the viruses in DOV, and all of them were classified as unknown Riboviria (Fig. S1).

Compared with the extensively studied marine DNA viruses, oyster-related virus investigations have focused mainly on transcriptomic data and RNA viruses (Rosani et al., 2017, Rosani et al., 2019; Zhang et al., 2021), thus missing the more ubiquitous marine DNA viruses. Ostreid herpesvirus is the most extensively studied DNA viral pathogen for oysters and many other aquaculture bivalves (De Lorgeril et al., 2018; Gao et al., 2018; Rosani \& Venier, 2017; Renault et al., 2000; Farley et al., 1972). We found that huge numbers of DNA viruses dominated at all the sampling sites (Fig. 1), which indicates the importance of DNA viruses in this unique habitat.

Viruses in order Caudovirales dominated the oyster virome (Figs. 2, S1), just as they dominate the public dataset and culture collections (Kauffman et al., 2018). Siphoviridae (28.5\%-30.61\%), Podoviridae $(13.46 \%-42.52 \%)$, and Myoviridae $(18.36 \%-29.61 \%)$ were the top three Caudovirales families in the DOV (Fig. 2A-C). Considering the primary bias of MDA, circular ssDNA viruses (including Microviridea and Circoviridea) accounted for only $2.23 \%$ of all the viruses (Fig. S1), which means their diversity is lower than the diversity of the dsDNA viruses in DOV. 
BLAST-based taxonomy of short contigs has limited accuracy (Jiang et al., 2011), and a large proportion of them (34.88\%) could not be assigned at the family level (Fig. S1). In view of this, PhaGCN was used and successfully classified 6,362 out of 8,760 large vOTUs ( $\geq 10 \mathrm{~kb}$ ) (Fig. 2B), which exceeded the number classified by vContact2 (214/8,760) (Fig. 2C), and the unassigned vOTUs decreased to $11.46 \%$ (Fig. 2D). Impressively, the DOV nodes (vOTUs) accounted for $74.58 \%$ of the total nodes, whereas the RefSeq nodes account for only $25.42 \%$ in the vContact2 network (Fig. 2E), indicating that current knowledge about the ocean virosphere is far from sufficient.

\subsection{Near-complete viral genomes}

A total of 3,473 viral contigs with $>90 \%$ genomic completeness (including 27 RNA viral genomes) were identified (Figs. 3, S2, Table S2). The genomes were 1,206-60,277 bp long, and the GC content was $24.74 \%-65.70 \%$ (Fig. S2). The encoded proteins shared only $0 \%-40 \%$ identity with known viral proteins (Fig. 3), which again indicated that most of the genomes represented new viral categories. Only 16 of them clustered with nonredundant reference genomes of CheckV with $95 \%$ average nucleotide identity and $70 \%$ alignment fraction of contigs. We considered both unknown and unclassified sequences $(67.1 \%$ $(2,330)$ of the total) as representing novel viruses at the family level (Table S2).

The classified genomes belonged to at least 11 DNA virus families. Those in order Caudovirales included Podoviridae (45), Sipoviridae (43), Myoviridae (14), and Autograhpiviridae (8) (Fig. S2). Circoviridae (order Cirlivirales) and Microviridae (order Petitvirales) were the most abundant families, accounting for $11.27 \%$ (399) and $6.98 \%$ (247) of the classified genomes, respectively (Fig. 3, Table S2). Moreover, in the viral proteomic phylogenetic tree, circovirus-related branches were widely dispersed and mixed with unannotated branches (Fig. S2), implying that many putative circovirus clades are yet to be identified.

\subsection{Oyster-related circoviruses}

Circoviridae have been commonly found in some virome studies, especially those based on the MDA method; however, most of the samples analyzed in these studies were environmental samples. Finding so many circovirus-like genomes in oysters was quite unexpected because all currently known hosts of circoviruses are in clade Bilateria in kingdom Animalia (Virus-Host Database, May 2021). Although this finding suggests that the circoviruses in oysters were mostly hosted by oysters, it is unlikely that other marine microorganisms commonly host circoviruses. Furthermore, we used the circovirus replicase protein sequences recorded by the International Committee on Taxonomy of Viruses (ICTV) as queries, and mined out 1,390 and 8,763 nearly complete circovirus-related replicase protein sequences from NCBI $\mathrm{nr}$ and DOV respectively by iterative BlastP searches. Similarity clustering of the identified replicase protein sequences (Fig. S3) shows that the circovirus-related sequences are very diverse. With the exception of two standard Circoviridae genera, Circovirus and Cyclovirus, which have been recorded by the ICTV, most of the other clusters contain sequences that have not been clearly classified (Fig. S3). Among them, the sequences from DOV accounted for $86.3 \%$ (6.3 times the percentage from NCBI nr) and 
were widely distributed and present in all the clusters. Some clusters even contained only DOV sequences, which indicates that these sequences may be unique to oysters (Fig. S3).

We also constructed a phylogeny tree (Fig. 4) using the replicase sequences that clustered with the circoviruses and cycloviruses (Fig. S3). The results showed that most of the replicase sequences from DOV were on an independent branch that was separate from the Circovirus and Cyclovirus branches, and distant from the branches of contaminant sequences (excluding the possibility of reagent contamination). We considered that these replicase sequences from DOV represented a new oyster- or bivalve-specific genus under Circoviridae, and temporarily named it Crasscircovirus (Fig. 4). Five of the DOV sequences were scattered in different Circovirus and Cyclovirus branches (Fig. 4). These findings suggest that oysters (and possibly bivalves) may be hotspots of circoviruses. Whether these circoviruses are pathogens or live as symbionts in oyster hosts, and whether they will spill-over to other marine animals like coronavirus in bats need further study (Van Brussel and Holmes, 2021).

\subsection{RNA viruses versus DNA viruses}

Most previous virome studies focused only on DNA or RNA viruses. Quantitatively comparing the diversity and abundance among RNA and DNA viruses in real environments will likely be very interesting (Holmes, E. C., 2011; Zhu et al., 2021; Steward et al., 2013). However, so that we could compare the results, in this study, we used various targeted amplifications to compare DNA and RNA viruses in the same sample separately (WGA and WTA) or simultaneously (RT-WGA).

Firstly, our study shows different amplification strategies can efficiently target different genomes, because the vOTUs of RNA viruses in the WTA libraries significantly outnumber those in the WGA libraries, and vice versa for the DNA viruses (Fig. S4A, B). Secondly, it seems to be common that the diversity of DNA viruses in nature and public databases is higher than the diversity of RNA viruses (Roux et al., 2021; Rosario et al., 2018; Levin et al., 2017). Although the differences in a-diversity indexes of WGA and WTA libraries were not significant, similar rules were still observed (Fig. S4D-F), which is consistent with previous obversions (Figs. 1C, S1). However, further studies are needed to confirm the conclusion that the diversity of DNA viruses is higher than that of RNA viruses. Furthermore, the extremely high mutation rates of RNA genomes challenged their detection recall of alignment-based annotations (Holmes, E. C., 2009; Shi et al., 2016), and the instability of RNA genomes and potential amplification bias further complicated the comparisons.

Notably, although the diversity of RNA viruses seemed low, their abundance in the WTA libraries was not low, reaching nearly $100 \%$ in some libraries (Fig. S5) where no active transcription products of DNA virus were detected. Similar results were not observed in the RT-WGA libraries (Fig. S5). The samples that contained a high proportion of RNA viruses need further investigation to determine which kinds of RNA viruses are dominant in these samples, and to understand why RNA and DNA viruses seem to occupy different niches and have different lifestyles. To compare the activity of RNA and DNA viruses in the 
same sample more objectively and effectively avoid the influence from host cells, metatranscriptome analysis may be an appropriate method.

\subsection{Viral communities}

Viral community studies can help determine whether the viruses enriched in oysters can be regarded as an organic whole, similar to viruses in the marine environment, or are simply a random and accidental assembly, as well as whether the community can respond to external influences. Although MDA introduces bias by prioritizing circular ssDNA genome (Binga et al., 2008), and this may have led to the $>80 \%$ abundance of circular ssDNA virus in several libraries in this study (Fig. S4). But Parras-Moltó et al. (2018) found that ordination plots based on dissimilarities among vOTU profiles showed perfect overlapping of related amplified and unamplified viromes and strong separation from unrelated viromes, which showed that MDA can be used for community studies.

We firstly evaluated the correlation among various community parameters, including the vOTU counts, the ratio of viral reads, varies diversity indexes, and the quantity and quality of sequencing reads (Fig. S6). The a-diversities correlated well among three community deciphering approaches (based on the OTU and AMG reference datasets) (Fig. S6), which indicates that the methodologies we used for community analysis verified each other. Secondly, as we expected, targeted amplification plays a decisive role in the virus community (Fig. 5A), and this was further verified by the community results based on reference genomes (Fig. 5B). Besides the amplification, the obviously different virus abundance patterns, as shown by the heatmap (Fig. 5C) and the F-value ranks (Fig. 5A), showed prominent differences between tissue groups. Even in an open circulatory system, the virus community in the tissue submerged by hemolymph was quite different from that in the hemolymph itself, which shows that different host tissues had a selective effect on the viruses.

Importantly, although the influence of health status, sampling sites, and sampling times on the whole community did not seem to be significant (Fig. 5A, low F-valve), we still found there were significant differences in both the $a$ - and $\beta$-diversity (NMDS) between all healthy and diseased samples (Fig. S7A, C). The a-diversity of moribund groups was relatively high, perhaps indicating that the decrease of immunity caused by diseases leads to an increase of opportunistic pathogens and their bacteriophages in the host. However, the expected differences between moribund and healthy groups were not detected in the parallel cohorts (Fig. S7B, C).

Geographical origin (sampling site) also substantially influenced the community. Samples from the same location tended to aggregate, and significant differences in a-diversity were observed from the WGA and WTA groups separately (Figure S8). The influence of the habitat on the microbiome of the host has been reported in many animals (Ge et al., 2021; Krotman et al., 2020; Sandri et al., 2020; Su et al., 2020), and environmental variations may be one of the reasons for the differences (Oetama et al., 2016). However, unlike freely swimming fish, oysters are sedentary and filter large volumes of the surrounding water daily (Bedford, 1978; Olalemi et al., 2016). The influence of site on the viromic community was weaker than 
that of the time point (Fig. 5A, lower F-value), and this was also reflected in the proportion of unique vOTUs (i.e., those that were detected only in one group) (Fig. S9). The relatively high proportion of unique vOTUs in the time period groups implies that viral communities are dynamic with time, and the low proportion of unique vOTUs indicates that viruses actively communicate among locations. However, because of the limited sample number and the diversity of host species, these results need further verification.

\subsection{Auxiliary metabolic genes (AMGs)}

Viruses play essential roles in metabolic regulation in the marine ecosystem (Suttle, 2005; Breitbart et al., 2012; Breitbart et al., 2018). Like marine viruses, a large number $(9,091)$ of AMGs were identified from DOV. They were assigned to 12 KEGG (Kyoto Encyclopedia of Genes and Genomes) pathways, and 98 pathways (Table S3). Among them, pathways associated with the metabolism of cofactors and vitamins, amino acids, energy, and carbohydrates were significantly enriched (Fig. S10A), which is similar to the results obtained for other marine viromes (Castelán-Sánchez et al., 2020; Hurwitz et al., 2016; Hurwitz et al., 2013). The previous finding that viruses with large genomes tend to encode more AMGs than viruses with small genomes and provide ecological functions beyond sustaining basic infection and proliferation (Jiang et al., 2020) is supported by the results shown in Fig. S10B. Importantly, the AMG community (Fig. S10C) showed consistency with the vOTU community (Fig. S10D), and the richness and Shannon index showed positive correlations between the two communities (Figs. S6, S10E, S10F). These findings indicate that the oyster viromic function was closely related to that of the species community. Although it is difficult to know which of them is the cause and which is the result, this discovery provides clues that can help further understand the ecological function of the virome in oysters.

\section{Conclusions}

Here we report a comprehensive viromic dataset (DOV) with high resolution that provides a new resource for studying and understanding the marine virome. We have shown from multiple aspects, including reads recruitment, vOTUs, high-quality virus genomes, and circovirus-related replicases, that oysters undoubtedly harbour a large, diverse, and unique array of viruses, which are very different from viruses in the ocean or any other habitat. Oysters can be considered as repositories and transmission hotspots of marine viruses, which may be caused by its filter-feeding lifestyle and high density of natural population. Notably, the viral communities in oysters are not random but well organized, and can respond to changes in host tissues and health state, and in the external environment at both compositional and functional levels. Further studies on the viral community structure and function of bivalves will greatly contribute to an understanding of their role in coastal microbiome regulation, in disease transmission, and in protecting and restoring coastal ecosystems.

\section{Declarations}




\section{Funding}

This project was supported by the Natural Science Foundation of China (nos. 31972847 and 32172955 ) to Jiang JZ and Duan M; Financial Fund of the Ministry of Agriculture and Rural Affairs, P. R. of China (NHYYSWZZZYKZX2020) to Zhang DC; the Central Public-Interest Scientific Institution Basal Research Fund, CAFS (nos. 2020TD42 and 2021SD05) to Jiang JZ; the Guangdong Provincial Special Fund for Modern Agriculture Industry Technology Innovation Teams (no. 2019KJ141) to Jiang JZ. The funders had no role in the study design, data collection and analysis, decision to publish, or manuscript preparation.

\section{Acknowledgment}

We thank Dr. Edward C. Holmes, Dr. Curtis A. Suttle, and Dr. Xu Kevin Zhong for their insightful comments and feedback. We thank Margaret Biswas, PhD, from Liwen Bianji (Edanz) (www.liwenbianji.cn/) for editing the English text of a draft of this manuscript.

\section{Ethics approval and consent to participate}

Not applicable.

\section{Consent for publication}

Not applicable.

\section{Competing interests}

The authors declare that they have no competing interests.

\section{Author details}

a Key Laboratory of South China Sea Fishery Resources Exploitation and Utilization, Ministry of Agriculture, South China Sea Fisheries Research Institute, Chinese Academy of Fishery Sciences, Guangzhou 510300, Guangdong, China. ${ }^{b}$ State Key Laboratory of Freshwater Ecology and Biotechnology, Institute of Hydrobiology, Chinese Academy of Sciences, Wuhan 430072, Hubei, China. ${ }^{c}$ College of Fisheries and Life Science, Shanghai Ocean University, Shanghai 201306, China. ${ }^{d}$ Guangdong Magigene Biotechnology Co., Ltd, Guangzhou 510000, Guangdong, China 
e School of Medicine, Sun Yat-sen University, Shenzhen 518107, Guangdong, China. ${ }^{f}$ Tianjin Agricultural University, Tianjin 300384, China. ${ }^{9}$ Shenzhen Fisheries Development Research Center, Shenzhen 518067 , Guangdong, China. ${ }^{\mathrm{h}}$ Bureau of Agriculture and Rural Affairs of Conghua District, Guangzhou 510925,

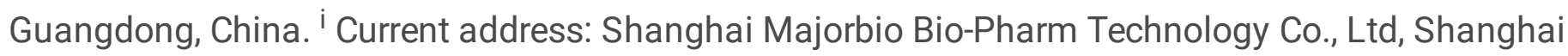
201203, China.

\section{Authors' contributions}

JJZ: Conceptualization, Methodology, Validation, Formal analysis, Investigation, Resources, Data Curation, Visualization, Writing - Original Draft, Writing - Review \& Editing, Supervision, Project administration, Funding acquisition; FYF: Validation, Formal analysis, Investigation, Resources, Data Curation, Writing - Original Draft, Visualization; WHY: Formal analysis, Investigation, Resources, Data Curation; ZP and LM: Formal analysis, Investigation, Visualization; YLL and GYX: Investigation, Resources; JT and SM: Writing - Review \& Editing; YT, LJ, YLT, SSK, and WM: Resources; DM and ZDC: Conceptualization, Methodology, Resources, Supervision, Project administration, Funding acquisition. All authors read and approved the final manuscript.

\section{Availability of data and materials}

The data set supporting the results of this article has been deposited in the Genome Sequence Archive (GSA) under BioProject accession code PRJCA007058[https://ngdc.cncb.ac.cn/gsub/submit/bioproject/subPR0010366/overview].

\section{References}

1. Andrade KR, Boratto PP, Rodrigues FP, Silva LC, Dornas FP, Pilotto MR et al. Oysters as hot spots for mimivirus isolation. Arch Virol. 2015;160(2):477-82.

2. Asplund M, Kjartansdóttir KR, Mollerup S, Vinner L, Fridholm H, Herrera JA, et al. Contaminating viral sequences in high-throughput sequencing viromics: a linkage study of 700 sequencing libraries. Clin Microbiol Infect. 2019;25(10):1277-85.

3. Bar-On YM, Phillips R, Milo R. The biomass distribution on Earth. Proc Natl Acad Sci U S A. 2018;115(25):6506-6511.

4. Bedford AJ, Williams G, Bellamy AR. Virus accumulation by the rock oyster Crassostrea glomerata. Appl Environ Microbiol. 1978;35(6):1012-8.

5. Berg G, Rybakova D, Fischer D, Cernava T, Vergès MC, Charles T, et al. Microbiome definition revisited: old concepts and new challenges. Microbiome. 2020;8:103. https://doi.org/10.1186/s40168020-00875-0 
6. Binga EK, Lasken RS, Neufeld JD. Something from (almost) nothing: the impact of multiple displacement amplification on microbial ecology. ISME J. 2008;2(3):233-241.

7. Breitbart M, Bonnain C, Malki K, Sawaya NA. Phage puppet masters of the marine microbial realm. Nat Microbiol. 2018;3(7):754-766. https://doi.org/10.1038/s41564-018-0166-y

8. Breitbart M. Marine viruses: truth or dare. Ann Rev Mar Sci. 2012;4:425-48.

9. Camarillo-Guerrero LF, Almeida A, Rangel-Pineros G, Finn RD, Lawley TD. Massive expansion of human gut bacteriophage diversity. Cell. 2021;184(4):1098-1109.e9.

10. Brum JR, Ignacio-Espinoza JC, Roux S, Doulcier G, Acinas SG, Alberti A, ... \& Sullivan MB. Patterns and ecological drivers of ocean viral communities. Science, 2015;348(6237).

11. Castelán-Sánchez HG, Meza-Rodríguez PM, Carrillo E, Ríos-Vázquez DI, Liñan-Torres A, Batista-García RA, et al. The microbial composition in circumneutral thermal springs from Chignahuapan, Puebla, Mexico reveals the presence of particular sulfur-oxidizing bacterial and viral communities. Microorganisms. 2020;8(11):1677. https://doi.org/10.3390/microorganisms8111677

12. de Lorgeril J, Lucasson A, Petton B, Toulza E, Montagnani C, Clerissi C, et al. Immune-suppression by OsHV-1 viral infection causes fatal bacteraemia in Pacific oysters. Nat Commun. 2018;9(1):4215.

13. Fan X, Yang C, Li W, Bai X, Zhou X, Xie H, et al. SMOOTH-seq: single-cell genome sequencing of human cells on a third-generation sequencing platform. Genome Biol. 2021;22(1):195.

14. Farley CA, Banfield WG, Kasnic G Jr, Foster WS. Oyster herpes-type virus. Science. 1972;178(4062):759-60.

15. Gao F, Jiang JZ, Wang JY, Wei HY. Real-time quantitative isothermal detection of Ostreid herpesvirus1 DNA in Scapharca subcrenata using recombinase polymerase amplification. J Virol Methods. 2018;255:71-75.

16. Ge Y, Jing Z, Diao Q, He JZ, Liu YJ. Host species and geography differentiate honeybee gut bacterial communities by changing the relative contribution of community assembly processes. mBio. 2021;12(3):e0075121.

17. Gregory AC, Zayed AA, Conceição-Neto N, Temperton B, Bolduc B, Alberti A, et al. Marine DNA viral macro- and microdiversity from Pole to Pole. Cell. 2019;177(5):1109-1123. https://doi.org/10.1016/j.cell.2019.03.040

18. Guo J, Bolduc B, Zayed AA, Varsani A, Dominguez-Huerta G, Delmont TO, et al. VirSorter2: a multiclassifier, expert-guided approach to detect diverse DNA and RNA viruses. Microbiome. 2021;9(1):37. https://doi.org/10.1186/s40168-020-00990-y

19. Handley SA, Virgin HW. Drowning in Viruses. Cell. 2019;177(5):1084-1085.

20. Holmes EC. Reagent contamination in viromics: all that glitters is not gold. Clin Microbiol Infect. 2019;25(10):1167-1168.

21. Holmes EC. The Evolution and Emergence of RNA Viruses. Oxford Univ Press. 2009.

22. Holmes EC. What does virus evolution tell us about virus origins? J Virol. 2011;85(11):5247-51. 
23. Hosono S, Faruqi AF, Dean FB, Du Y, Sun Z, Wu X, et al. Unbiased whole-genome amplification directly from clinical samples. Genome Res. 2013;13(5):954-64.

24. Hurwitz BL, Hallam SJ, Sullivan MB. Metabolic reprogramming by viruses in the sunlit and dark ocean. Genome Biol. 2013;14(11):R123. https://doi.org/10.1186/gb-2013-14-11-r123

25. Hurwitz BL, U'Ren JM. Viral metabolic reprogramming in marine ecosystems. Curr Opin Microbiol. 2016;31:161-168.

26. Huson DH, Beier S, Flade I, Górska A, El-Hadidi M, Mitra S, et al. MEGAN Community Edition Interactive exploration and analysis of large-scale microbiome sequencing data. PLoS Comput Biol. 2016;12(6): e1004957.

27. Jiang JZ, Zhang W, Guo ZX, Cai CC, Su YL, Wang RX, et al. Functional annotation of an expressed sequence tag library from Haliotis diversicolor and analysis of its plant-like sequences. Mar Genomics. 2011;4(3):189-96.

28. Jiang T, Guo C, Wang M, Wang M, Zhang X, Liu Y, et al. Genome Analysis of Two Novel Synechococcus Phages That Lack Common Auxiliary Metabolic Genes: Possible Reasons and Ecological Insights by Comparative Analysis of Cyanomyoviruses. Viruses. 2020;12(8):800. https://doi.org/10.3390/v12080800

29. Kauffman KM, Hussain FA, Yang J, Arevalo P, Brown JM, Chang WK, et al. A major lineage of nontailed dsDNA viruses as unrecognized killers of marine bacteria. Nature. 2018;554(7690):118-122. https://doi.org/10.1038/nature25474

30. Kieft K, Zhou Z, Anantharaman K. VIBRANT: automated recovery, annotation and curation of microbial viruses, and evaluation of viral community function from genomic sequences. Microbiome. 2020;8(1):90. https://doi.org/10.1186/s40168-020-00867-0

31. Krotman Y, Yergaliyev TM, Alexander Shani R, Avrahami Y, Szitenberg A. Dissecting the factors shaping fish skin microbiomes in a heterogeneous inland water system. Microbiome. 2020;8(1):9.

32. Levin RA, Voolstra CR, Weynberg KD, van Oppen MJ. Evidence for a role of viruses in the thermal sensitivity of coral photosymbionts. ISME J. 2017;11(3):808-812. https://doi.org/10.1038/ismej.2016.154

33. Li Y, Fu X, Ma J, Zhang J, Hu Y, Dong W, et al. Altered respiratory virome and serum cytokine profile associated with recurrent respiratory tract infections in children. Nat Commun. 2019;10(1):2288.

34. Liu P, Chen W, Chen JP. Viral metagenomics revealed Sendai virus and Coronavirus infection of Malayan pangolins (Manis javanica). Viruses. 2019;11(11):979.

35. Nayfach S, Camargo AP, Schulz F, Eloe-Fadrosh E, Roux S, Kyrpides NC. CheckV assesses the quality and completeness of metagenome-assembled viral genomes. Nat Biotechnol. 2021;39(5):578-585.

36. Nayfach S, Páez-Espino D, Call L, Low SJ, Sberro H, Ivanova NN, et al. Metagenomic compendium of 189,680 DNA viruses from the human gut microbiome. Nat Microbiol. 2021;6(7):960-970. https://doi.org/10.1038/s41564-021-00928-6

37. Newell DG, Koopmans M, Verhoef L, Duizer E, Aidara-Kane A, Sprong H, et al. Food-borne diseases the challenges of 20 years ago still persist while new ones continue to emerge. Int J Food Microbiol. 
2010;139(Suppl 1):S3-15.

38. Nouri S, Matsumura EE, Kuo YW, Falk BW. Insect-specific viruses: from discovery to potential translational applications. Curr Opin Virol. 2018;33:33-41.

39. Oetama VSP, Hennersdorf P, Abdul-Aziz MA, Mrotzek G, Haryanti H, Saluz HP. Microbiome analysis and detection of pathogenic bacteria of Penaeus monodon from Jakarta Bay and Bali. Mar Pollut Bull. 2016;110(2):718-725.

40. Olalemi A, Baker-Austin C, Ebdon J, Taylor H. Bioaccumulation and persistence of faecal bacterial and viral indicators in Mytilus edulis and Crassostrea gigas. Int J Hyg Environ Health. 2016;219(7 Pt A):592-598.

41. Paez-Espino D, Eloe-Fadrosh EA, Pavlopoulos GA, Thomas AD, Huntemann M, Mikhailova N, et al. Uncovering Earth's virome. Nature. 2016;536(7617):425-30. https://doi.org/10.1038/nature19094

42. Pan X, Durrett RE, Zhu H, Tanaka Y, Li Y, Zi X, et al. Two methods for full-length RNA sequencing for low quantities of cells and single cells. Proc Natl Acad Sci U S A. 2013;110(2):594-9.

43. Parras-Moltó M, Rodríguez-Galet A, Suárez-Rodríguez P, López-Bueno A. Evaluation of bias induced by viral enrichment and random amplification protocols in metagenomic surveys of saliva DNA viruses. Microbiome. 2018;6(1):119.

44. Patro R, Duggal G, Love MI, Irizarry RA, Kingsford C. Salmon provides fast and bias-aware quantification of transcript expression. Nat Methods. 2017;14(4):417-419.

45. Picher ÁJ, Budeus B, Wafzig O, Krüger C, García-Gómez S, Martínez-Jiménez MI, et al. TruePrime is a novel method for whole-genome amplification from single cells based on TthPrimPol. Nat Commun. 2016;7:13296.

46. Polson SW, Wilhelm SW, Wommack KE. Unraveling the viral tapestry (from inside the capsid out). ISME J. 2011;5(2):165-8.

47. Porter AF, Cobbin J, Li C, Eden JS, Holmes EC. Metagenomic identification of viral sequences in laboratory reagents. bioRxiv. 2021. https://doi.org/10.1101/2021.09.10.459871

48. Powell D, Subramanian S, Suwansa-Ard S, Zhao M, O'Connor W, Raftos D, et al. The genome of the oyster Saccostrea offers insight into the environmental resilience of bivalves. DNA Res. 2018;25(6):655-665.

49. Renault T, Le Deuff RM, Chollet B, Cochennec N, Gérard A. Concomitant herpes-like virus infections in hatchery-reared larvae and nursery-cultured spat Crassostrea gigas and Ostrea edulis. Dis Aquat Organ. 2000;42(3):173-183.

50. Renault T, Novoa B. Viruses infecting bivalve molluscs. Aquat Living Resour. 2004;17(4):397-409.

51. Rosani U, Gerdol M. A bioinformatics approach reveals seven nearly-complete RNA-virus genomes in bivalve RNA-seq data. Virus Res. 2017;239:33-42.

52. Rosani U, Shapiro M, Venier P, Allam B. A needle in a haystack: tracing bivalve-associated viruses in high-throughput transcriptomic data. Viruses. 2019;11(3):205. 
53. Rosani U, Venier P. Oyster RNA-seq Data Support the Development of Malacoherpesviridae Genomics. Front Microbiol. 2017;8:1515.

54. Rosario R, Fierer N, Miller S, Luongo J, Breitbart M. Diversity of DNA and RNA viruses in indoor air as assessed via metagenomic sequencing. Environ Sci Technol. 2018;52(3):1014-1027.

55. Roux S, Páez-Espino D, Chen IA, Palaniappan K, Ratner A, Chu K, et al. IMG/VR v3: an integrated ecological and evolutionary framework for interrogating genomes of uncultivated viruses. Nucleic Acids Res. 2021;49(D1):D764-D775. https://doi.org/10.1093/nar/gkaa946

56. Sandri C, Correa F, Spiezio C, Trevisi P, Luise D, Modesto M, et al. Fecal microbiota characterization of seychelles giant tortoises (Aldabrachelys gigantea) living in both wild and controlled environments. Front Microbiol. 2020;11:569249.

57. Scanes E, Parker LM, Seymour JR, Siboni N, King WL, Danckert NP, et al. Climate change alters the haemolymph microbiome of oysters. Mar Pollut Bull. 2021;164:111991.

58. Shang J, Jiang J, Sun Y. Bacteriophage classification for assembled contigs using graph convolutional network. Bioinformatics. 2021;37(Suppl_1):i25-i33.

59. Shi M, Lin XD, Chen X, Tian JH, Chen LJ, Li K, et al. The evolutionary history of vertebrate RNA viruses. Nature. 2018;556(7700):197-202. https://doi.org/10.1038/s41586-018-0012-7

60. Shi M, Lin XD, Tian JH, Chen LJ, Chen X, Li CX, et al. Redefining the invertebrate RNA virosphere. Nature. 2016;540(7634):539-543. https://doi.org/10.1038/nature20167

61. Stepanauskas R, Fergusson EA, Brown J, Poulton NJ, Tupper B, Labonté JM, et al. Improved genome recovery and integrated cell-size analyses of individual uncultured microbial cells and viral particles. Nat Commun. 2017;8(1):84.

62. Steward GF, Culley Al, Mueller JA, Wood-Charlson EM, Belcaid M, Poisson G. Are we missing half of the viruses in the ocean? ISME J. 2013;7(3):672-9. https://doi.org/10.1038/ismej.2012.121

63. Su S, Munganga BP, Du F, Yu J, Li J, Yu F, et al. Relationship between the fatty acid profiles and gut bacterial communities of the chinese mitten crab (Eriocheir sinensis) from ecologically different habitats. Front Microbiol. 2020;11:565267.

64. Suttle CA. Viruses in the sea. Nature. 2005;437(7057):356-61.

65. Van Brussel K, Holmes EC. Zoonotic disease and virome diversity in bats. Curr Opin Virol. 2021;23(52):192-202.

66. Wei HY, Huang S, Wang JY, Gao F, Jiang JZ. Comparison of methods for library construction and short read annotation of shellfish viral metagenomes. Genes Genom. 2018a;40(3):281-288. https://doi.org/10.1007/s13258-017-0629-1

67. Wei HY, Huang S, Yao T, Gao F, Jiang JZ, Wang JY. Detection of viruses in abalone tissue using metagenomics technology. Aquaculture Research. 2018b;49(8):2704-2713.

68. Wolf YI, Silas S, Wang Y, Wu S, Bocek M, Kazlauskas D, et al. Doubling of the known set of RNA viruses by metagenomic analysis of an aquatic virome. Nat Microbiol. 2020;5(10):1262-1270. 
69. Yao Z, Zou C, Peng N, Zhu Y, Bao Y, Zhou Q, et al. Virome identification and characterization of Fusarium sacchari and F. andiyazi: causative agents of Pokkah Boeng disease in sugarcane. Front Microbiol. 2020;11:240.

70. Zhang G, Fang X, Guo X, Li L, Luo R, Xu F, et al. The oyster genome reveals stress adaptation and complexity of shell formation. Nature. 2012;490(7418):49-54. https://doi.org/10.1038/nature11413

71. Zhang YY, Chen Y, Wei X, Cui J. Viromes in marine ecosystems reveal remarkable invertebrate RNA virus diversity. Sci China Life Sci. 2021. https://doi.org/10.1007/s11427-020-1936-2

72. Zuo T, Liu Q, Zhang F, Yeoh YK, Wan Y, Zhan H, et al. Temporal landscape of human gut RNA and DNA virome in SARS-CoV-2 infection and severity. Microbiome. 2021;9(1):91.

\section{Figures}

\section{Figure 1}

Overview of the Dataset of Oyster Virome (DOV). (A) De novo assembly and annotation pipeline. (B) Sankey diagram of the relationship among different batches and groups. The heights of the black vertical bars proportionally represent the number of viral contigs (vOTUs) assembled under teach group. (C) Rarefaction curves of the oyster viromic libraries. RT-WGA, reverse transcription and whole genome amplification; WGA, whole genome amplification; WTA whole transcriptome amplification. (D) Sampling site distribution map and the number of detected vOTUs from each site. The radius of the pie chart indicates the number of DNA, RNA, and unclassified vOTUs. (E) Mapping rates of viral reads in total clean reads. RefSeq, NCBI viral RefSeq genomes (release June 2019); GOV (release July 2020), Global Ocean Virome dataset; IMG/VR (release January 2018), a database of cultured and uncultured DNA viruses and

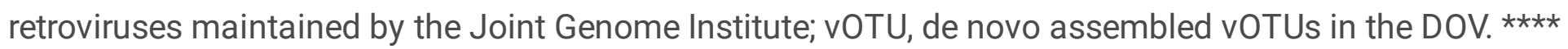
indicates $p<0.0001$ (Student's t-test between the three mapping rates).

\section{Figure 2}

Taxonomy classification of the Dataset of Oyster Virome (DOV) at the family level. (A-C) Pie charts showing the proportion of different viral families in the total viral contigs (vOTUs) longer than $800 \mathrm{bp}$. The vOTUs were classified using Diamond (v0.9.14) (A), and vOTUs longer than $10 \mathrm{~kb}$ were classified using PhaGCN (B) and vContact2 (C). The numbers in parentheses indicate the number of vOTU successfully classified/total number of vOTU. (D, E) vContact2 networks constructed with vOTUs and NCBI RefSeq viral genomes (release June 2019) longer than $10 \mathrm{~kb}$ showing they have the same topology. The colors of the nodes indicate different PhaGCN families (D), and their sources (E). n, total number of nodes in vContact2 networks. The percentage of each family or source in (D) is listed after corresponding legends. 


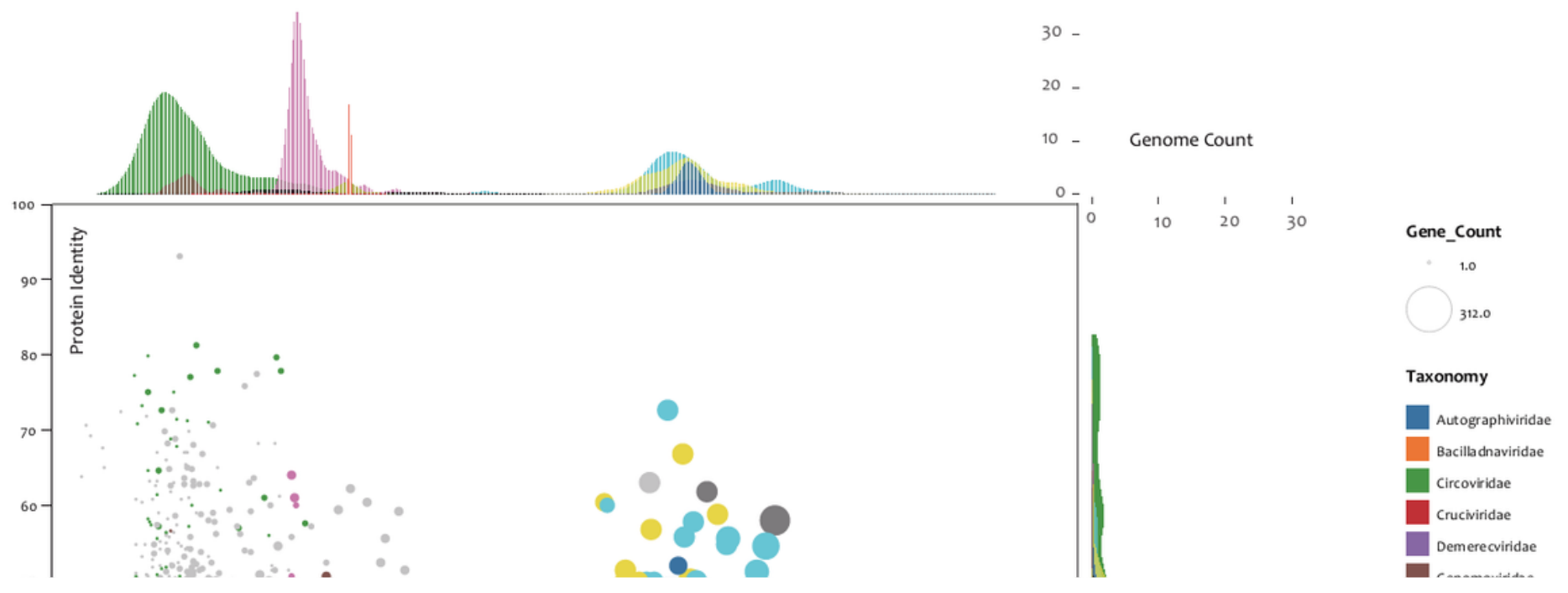

Figure 3

Scatter plot of complete and near-complete viral genomes in the Dataset of Oyster Virome (DOV). X-axis, log10 value of viral genomic length; Y-axis, protein identity between DOV genomes and CheckV reference genomes. The diameter of the circles indicates the gene count in the genomes. Colors indicate different viral families obtained by combining the PhaGCN and Diamond (v0.9.14.115) results. The density histograms parallel to the $\mathrm{X}$ - and $\mathrm{Y}$-axes show the distribution of the gene count for the corresponding viral genomes. 

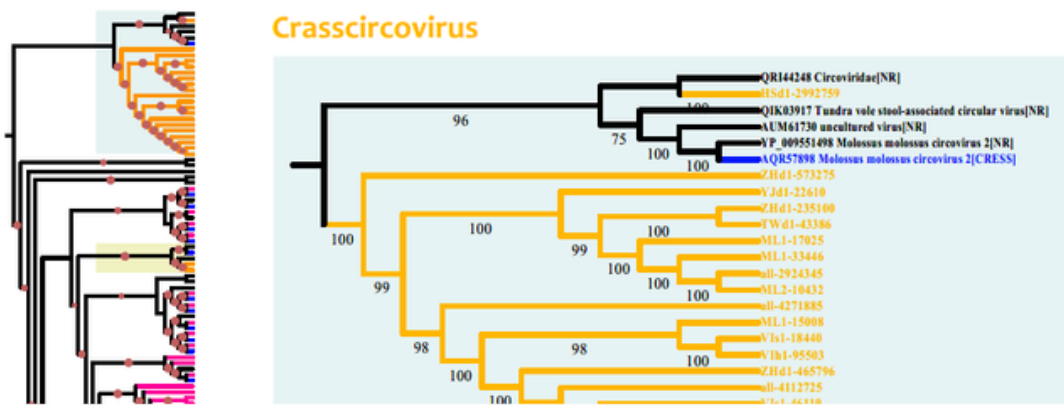

\section{Figure 4}

Phylogeny of replicase proteins of oyster related circoviruses. The large tree on the left shows the phylogeny of all proteins in the clusters of the two standard Circoviridae genera recorded by the International Committee on Taxonomy of Viruses (ICTV) (Fig. S3), namely Cyclovirus (green) and Circovirus (violet). The diameter of red dots on branches represents the bootstrap value, and only values above 70 are shown. Small trees on the right are enlarged branches from the tree on the left. Background 
colors indicate different viral genera: light blue, Crasscircovirus; yellow, Cyclovirus; pink, Circovirus. Colors of branches indicate data origins: orange, Dataset of Oyster Virome (DOV); grey, NCBI nr database; blue, CRESS viruses from Ashleigh et al. (2021); green, circoviruses from the ICTV; violet, cycloviruses from the ICTV; red, contaminant sequences from Asplund et al. (2019) and Ashleigh et al. (2021); black, other NCBI $\mathrm{nr}$ sequences.

\section{Figure 5}

Viral community in the Dataset of Oyster Virome (DOV). (A) Nonmetric multidimensional scaling (NMDS) analysis shows the clusters of DOV libraries according to amplification groups. Nonparametric multivariate analysis of variance (permanova) was used. RT-WGA, reverse transcription and whole genome amplification; WGA, whole genome amplification; WTA whole transcriptome amplification. (B) Procrustes analysis of NMDS coordinates of viral communities based on comparisons of reference genomes (RefSeq, GOV, and IMG/VR) and de novo assembled viral contigs (vOTUs). (C) Heatmap of DOV vOTUs. The vOTUs clustered by the Euclidean method and colored by the viral genome types (dsDNA, sSDNA, RNA, and unclassified) are shown on the Y-axis. The DOV libraries ordered by amplification strategy (WGA, RT_WGA, and WTA) and tissue origin (hemolymph and mixed tissue) are shown on the Xaxis.

\section{Supplementary Files}

This is a list of supplementary files associated with this preprint. Click to download.

- TableS1.xIsx

- TableS3.xIsx

- Tables2.xlsx

- DOVSupplementaryV3.docx 